\title{
Field Validation of a Rapid Recombinase Aided Amplification Assay for SARS-CoV-2 RNA at Customs - Zhejiang Province, China, January 2021
}

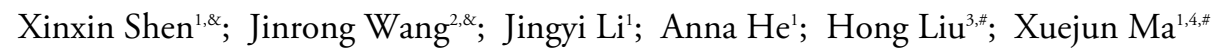

\begin{abstract}
Introduction: The best approach to preventing the importation of coronavirus disease 2019 (COVID-19) is enhancing the detection capacity at customs. The rapid detection is of utmost importance and therefore highly demanded.

Methods: We conducted a field validation study of a duplex real-time reverse transcription recombinaseaided amplification (RT-RAA) assay in Zhoushan and Hangzhou customs, in Zhejiang Province, China. The reverse transcriptase polymerase chain reaction (RTPCR) assay kit routinely used at customs was used in parallel, and the duration the two methods took to complete a specific number of samples was compared.

Results: Among 506 samples collected, RT-RAA results were consistent with the RT-PCR results. The sensitivity and specificity were $100 \%$, the total coincidence rate was $100 \%$, and the Kappa value was 1 $(P<0.05)$ for both methods. The RT-RAA kit took a significantly shorter time in testing the 20-200 samples than the RT-PCR kit.

Discussion: The RT-RAA detection method is more efficient and suitable for use at customs than RTPCR assay to realize rapid customs clearance of 200 or fewer samples.
\end{abstract}

\section{INTRODUCTION}

The coronavirus disease 2019 (COVID-19) pandemic has raised serious health and economic concerns worldwide (1). At present, China has effectively controlled the local spread of the disease. However, COVID-19 is still an active epidemic in many countries across the world (2-3). Therefore, preventing the importation of new COVID-19 strains from overseas and rebound of the domestic outbreaks are currently two major focus areas in China. The best approach to preventing the importation of COVID-19 from other countries is enhancing the detection capacity at customs where the detection accuracy and rapidity are highly demanded (4).

Reverse transcriptase polymerase chain reaction (RTPCR) is a commonly used technique at customs for diagnosing COVID-19 (5). However, this method is time-consuming and requires specialized equipment and skilled personnel (๑). The IgM or IgG-based assay has low sensitivity and requires a longer window period (7-8), making it unsuitable for early diagnosis, which is critical at customs. Previously, we conducted a multiple-center clinical evaluation of an ultrafast single-tube assay for severe acute respiratory syndrome coronavirus 2 (SARS-CoV-2) RNA. The method involved using a reverse transcription recombinase aided amplification (RT-RAA) assay. Notably, the detection was completed within 15 minutes at $39{ }^{\circ} \mathrm{C}$ using portable instruments after adding the extracted RNA samples (9-10). Our findings demonstrate that the RT-RAA assay has comparable sensitivity and specificity to the commercial RT-PCR kits and exhibits the distinctive advantages of simplicity and rapidity regarding operation and turnaround time. We validated the preceding RT-RAA assay by incorporating an internal control in the RAA system (11). The duplex RT-RAA kit was evaluated by the National Institutes for Food and Drug Control, China. According to the results, duplex RT-RAA kit had good specificity and the sensitivity of the duplex RT-RAA kit reached the range of 45 copies $/ \mathrm{mL}$ to 137 copies $/ \mathrm{mL}$. Therefore, we conducted a field validation study at Zhoushan and Hangzhou customs, in Zhejiang Province, China. To evaluate the specificity and sensitivity of the RT-RAA kit, the PCR assay kit routinely used at customs was used in parallel, and the duration the two methods took to complete a specific number of samples was compared. This study provides reference data for establishing a rapid on-site detection of SARS-CoV-2 RNA at customs. 


\section{MATERIALS AND METHODS}

\section{Samples}

A total of 506 nasopharyngeal swabs (273 samples in Hangzhou Customs and, 233 samples in Zhoushan Customs) were collected from visitors entering China between January 1, 2021, and January 25, 2021. The sample collection and preservation were performed according to the Guide of Laboratory Techniques for testing novel coronavirus infected pneumonia released by the National Health Commission of the People's Republic of China. After collection, the samples were inactivated at $56{ }^{\circ} \mathrm{C}$ for $30 \mathrm{~min}$ and stored at $-80{ }^{\circ} \mathrm{C}$. This study was approved by the institutional review committees of Hangzhou and Zhoushan Customs and was conducted as per the National Code of Ethics.

\section{Nucleic Acid Extraction}

Nucleic acid extraction was performed using the existing automated nucleic acid extractor that is routinely used at customs for detecting SARS-CoV-2 RNA. The specific nucleic acid extraction methods used at customs were summarized in Table 1. Total DNA/RNA was extracted from the samples using the methods outlined in Table 1, according to the manufacturers' instructions, and stored at $-80{ }^{\circ} \mathrm{C}$ until use.

SARS-CoV-2 RNA was detected from the clinical samples using the COVID-19 RT-RAA kit, according to the methods described in our previous report (10-11). Positive controls (recombinant plasmids) and negative controls (DNase-free water) were included in each run to ensure the reliability of the experimental results. The 6-carboxy-fluoescein (FAM) channel was used to detect the amplification of the target gene, whereas the Hexachloro fluorescein (HEX) channel was used to detect the amplification of the internal control gene. The results were considered to be positive when both channels were positive or when the FAM channel was positive, and the HEX channel was negative. When the FAM channel was negative, but the HEX channel was positive, the result was negative. When both channels were negative, the result was considered invalid, and the RT-RAA assay was redone. The time it took to analyze specific numbers of samples using a particular method was recorded.

\section{Analysis of Clinical Samples Using Reference RT-PCR Methods at Customs}

Clinical samples were assessed for the presence of SARS-CoV-2 viral RNA using commercial COVID-19 quantitative RT-PCR kits (Easy Diagnosis Biomedicine Co, Wuhan, China, and DAAN GENE, Guangzhou, China in ZhouShan Customs, Easy Diagnosis Biomedicine Co, Wuhan, China and, BioGerm Medical Technology, Shanghai, China in Hangzhou Customs), according to the manufacturers' instructions. RT-PCR was performed alongside the RT-RAA kits at the facility sites (Hangzhou and Zhoushan Customs), where the clinical samples were stored. All the PCR procedures were performed on an ABI 7500 Real-Time PCR Instrument (Applied Biosystems, Foster City, CA) provided by the local customs. The time required to analyze a specific number of samples using a given method was recorded.

\section{Comparing the Efficiency of RAA Versus RT-PCR for the Detection of SARS-COV-2 RNA}

The efficiencies of the COVID-19 nucleic acid detection kit (Fluorescence RT-RAA) and commercial RT-PCR nucleic acid detection kit were compared at the Hangzhou and Zhoushan customs. The parameters compared included the sensitivity, specificity, overall agreement rate, Kappa coefficient, and the time it took to complete $14,28,56,98$, and 196 samples using a specific method (12).

\section{Statistical Data Analysis}

IBM SPSS Statistics (version 21, IBM Corporation, NY, USA) was used to perform all the statistical

TABLE 1. Nucleic acid extraction methods used at the customs.

\begin{tabular}{ccccc}
\hline Manufacturer & Instrument model & Reagent & Extraction duration (min) & Institution \\
\hline Tianlong & GeneRotex 96 & Nucleic acid extraction kit & 24 & Hangzhou customs \\
Tianlong & NP968 & Nucleic acid extraction kit & 15 & Zhoushan customs \\
Tiangen & TGuide S32 & Total RNA extraction kit & 18 & Zhoushan customs \\
Bioperfectus & SSNP-2000A & Viral nucleic acid extraction kit & 23 & Zhoushan customs \\
\hline
\end{tabular}

Note: Detection of SARS-CoV-2 viral RNA in the clinical samples using RT-RAA kits.

Abbreviation: RT-RAA=real-time reverse transcription recombinase-aided amplification. 
analysis. The results were analyzed using Kappa test and paired-samples t test, and a $P$-value less than 0.05 was considered statistically significant.

\section{RESULTS}

Analytical results of RAA were compared to those of RT-PCR for SARS-CoV-2 at Hangzhou Customs and Zhoushan Customs. Among the 506 samples collected by the Hangzhou customs and Zhoushan customs, 16 were positive, whereas 490 were negative as detected using the RT-RAA kit (Table 2). These results were consistent with the RT-PCR results. The sensitivity and specificity were $100 \%$, the total coincidence rate was $100 \%$, and the Kappa value was $1(P<0.05)$ for both methods.

A time comparison was performed for the simultaneous detection of different numbers of specimens using RT-RAA and RT-PCR at Hangzhou Customs. The detection time of the RT-RAA kit was significantly shorter than that of the RT-PCR kit for samples less than 200, especially in the detection of samples $<100$ (Table 3).

\section{DISCUSSION}

The COVID-19 pandemic has seriously affected the physical and mental health of the world population. In addition, the pandemic situation has led to a significant reduction in economic development and social stability globally (13). Unfortunately, these circumstances are likely to persist for a long time before herd immunity can be achieved worldwide (14). As a forefront institution in the prevention of COVID-19 importation, China customs is still facing an enormous challenge, mainly because the rapid nucleic acid detection kits currently used are not suitable for SARS-CoV-2 on-site detection (15). Specifically, these kits are time-consuming and less sensitive. Therefore, there is an urgent need to develop more efficient techniques and products to fill in the deficiency of the existing ones.

Therefore, we demonstrate that the RT-RAA detection kit is accurate for detecting SARS-CoV-2 RNA in swab samples (10). This was verified by field testing of 506 samples (273 cases in Hangzhou customs and 233 cases in Zhoushan customs). Based on the testing results, the sensitivity and specificity of the RT-RAA detection kit was $100 \%$, which was consistent with the results obtained using RT-PCR detection kits. Notably, the RAA detection reagents and equipment (RAA-B6100 and RAA-F1620) matched different nucleic acid extraction devices/reagents currently used at customs. The nucleic acid extractors used at customs include Tianlong GeneRotex 96, Tianlong NP968, Tiangen TGuide S32, and Bioperfectus SSNP-2000A.

The most distinctive features of the RT-RAA detection kit were its rapidity and flexibility. According to the results obtained after analyzing 20-200 samples, the RT-RAA kit was more efficient and took a significantly shorter time than the RT-PCR kit (for example, $22 \mathrm{~min} v s .110 \mathrm{~min}$ for 14 samples, $112 \mathrm{~min}$ vs. 220 min for 98 samples). The RAA detection kit exhibits significant advantages over the RT-PCR kit, such as fast and flexible detection, and is, therefore, more suitable for use at customs clearance. Of note, it is mandatory to reanalyze clinical samples which turn positive after the initial test. In this case, the RT-RAA detection method is more applicable as it takes only 20 min compared to the RT-PCR method, which takes more than $90 \mathrm{~min}$.

The limitations of current RT-RAA detection kit were the moderate throughput (16 samples one run) and requirement of nucleic acid extraction, which will be addressed in the future to better serve the customs. Nevertheless, this study showed that the RT-RAA detection method in its current format was efficient and suitable for use at customs as an alternative to RTPCR assay. Therefore, we recommend that customs

TABLE 2. Results of SARS-CoV-2 analysis using RT-RAA versus RT-PCR detection kits (Hangzhou customs and Zhoushan customs).

\begin{tabular}{|c|c|c|c|c|c|c|c|}
\hline \multirow{2}{*}{$\begin{array}{l}\text { Results obtained using RT-RAA } \\
\text { nucleic acid detection kits }\end{array}$} & \multicolumn{3}{|c|}{$\begin{array}{c}\text { Results obtained using RT-PCR nucleic acid } \\
\text { detection kits }\end{array}$} & \multirow{2}{*}{$\begin{array}{l}\text { Sensitivity } \\
(\%)\end{array}$} & \multirow{2}{*}{$\begin{array}{l}\text { Specificity } \\
(\%)\end{array}$} & \multirow{2}{*}{ Kappa } & \multirow{2}{*}{$P$} \\
\hline & Positive & Negative & Total & & & & \\
\hline Positive & 16 & 0 & 16 & & & & \\
\hline Negative & 0 & 490 & 490 & 100 & 100 & 1 & $<0.05$ \\
\hline Total & 16 & 490 & 506 & & & & \\
\hline
\end{tabular}

Note: Time comparison of simultaneous detection of different numbers of specimens by RT-RAA and RT-PCR at Hangzhou customs. Abbreviations: RT-RAA=real-time reverse transcription recombinase-aided amplification; RT-PCR=reverse transcriptase polymerase chain reaction. 
TABLE 3. Comparison of the detection time between RT-RAA and RT-PCR detection kits.

\begin{tabular}{cccccc}
\hline Sample size (a) & $\mathbf{1 4}$ & $\mathbf{2 8}$ & $\mathbf{5 6}$ & $\mathbf{9 8}$ & $\mathbf{1 9 6}$ \\
\hline Detection time (RT-RAA), minutes & 22 & 37 & 67 & 112 & 202 \\
Detection time (RT-PCR), minutes & 110 & 110 & 110 & 220 & 330 \\
$P$ & & & $<0.05$ & &
\end{tabular}

Abbreviations: RT-RAA=real-time reverse transcription recombinase-aided amplification; RT-PCR=reverse transcriptase polymerase chain reaction.

adopt the RT-RAA detection method reported in the current study to realize rapid customs clearance of 200 or fewer samples. We anticipate that RT-RAA can be used to analyze 300 samples within 2 hours with more devices and personnel.

Conflicts of Interest: No conflict of interest declared.

Acknowledgements: Zhoushan and Hangzhou customs, Zhejiang Province, and Jiangsu Qitian Gene Biotechnology Co. Ltd.

Funding: Supported by grants from IVDC (2019HYDQNJJ03) and the key $\mathrm{R} \& \mathrm{D}$ projects in Zibo City (2020kj100011).

doi: $10.46234 / \mathrm{ccdcw} 2021.236$

* Corresponding authors: Hong Liu, liuhongseminar@hotmail.com; Xuejun Ma, maxj@ivdc.chinacdc.cn.

\footnotetext{
${ }^{1}$ National Institute for Viral Disease Control and Prevention, Chinese Center for Disease Control and Prevention, Beijing, China; 2 Shijiazhuang People's Hospital, Shijiazhuang, Hebei, China; ${ }^{3}$ Shandong Provincial Research Center for Bioinformatic Engineering and Technique, School of Life Sciences and Medicine, Shandong University of Technology, Zibo, Shandong, China; ${ }^{4}$ Center for Biosafety Mega-Science, Chinese Academy of Sciences, Wuhan, Hubei, China.

\& Joint first authors.
}

Submitted: August 25, 2021; Accepted: October 22, 2021

\section{REFERENCES}

1. Sharma A, Ahmad Farouk I, Lal SK. COVID-19: a review on the novel coronavirus disease evolution, transmission, detection, control and prevention. Viruses 2021;13(2):202. http://dx.doi.org/10.3390/v13 020202.

2. Salzberger B, Buder F, Lampl B, Ehrenstein B, Hitzenbichler F, Holzmann T, et al. Epidemiology of SARS-CoV-2. Infection 2021; 49(2):233 - 9. http://dx.doi.org/10.1007/s15010-020-01531-3.

3. Atzrodt CL, Maknojia I, McCarthy RDP, Oldfield TM, Po J, Ta KTL, et al. A guide to COVID-19: a global pandemic caused by the novel coronavirus SARS-CoV-2. FEBS J 2020;287(17):3633 - 50. http://dx. doi.org/10.1111/febs.15375.

4. Read JM, Bridgen JRE, Cummings DAT, Ho A, Jewell CP. Novel coronavirus 2019-nCoV (COVID-19): early estimation of epidemiological parameters and epidemic size estimates. Philos Trans Roy Soc B Biol Sci 2021;376(1829):20200265. http://dx.doi.org/ 10.1098/rstb.2020.0265.

5. Wikramaratna PS, Paton RS, Ghafari M, Lourenço J. Estimating the false-negative test probability of SARS-CoV-2 by RT-PCR. Eurosurveillance 2020;25(50):2000568. http://dx.doi.org/10.2807/ 1560-7917.ES.2020.25.50.2000568.

6. Xue GH, Li SL, Zhang WW, Du B, Cui JH, Yan C, et al. Reversetranscription recombinase-aided amplification assay for rapid detection of the 2019 novel coronavirus (SARS-CoV-2). Anal Chem 2020; 92(14):9699 - 705. http://dx.doi.org/10.1021/acs.analchem.0c01032.

7. Padoan A, Cosma C, Sciacovelli L, Faggian D, Plebani M. Analytical performances of a chemiluminescence immunoassay for SARS-CoV-2 IgM/IgG and antibody kinetics. Clin Chem Lab Med 2020;58(7): 1081 - 8. http://dx.doi.org/10.1515/cclm-2020-0443.

8. Safiabadi Tali SH, LeBlanc JJ, Sadiq Z, Oyewunmi OD, Camargo C, Nikpour B, et al. Tools and techniques for severe acute respiratory syndrome coronavirus 2 (SARS-CoV-2)/COVID-19 detection. Clin Microbiol Rev 2021;34(3):e00228 - 20. http://dx.doi.org/10.1128/ CMR.00228-20.

9. Shahrajabian MH, Sun WL, Cheng Q. Different methods for molecular and rapid detection of human novel coronavirus. Curr Pharm Des 2021;27(25):2893 - 903. http://dx.doi.org/10.2174/1381612827 666210604114411.

10. Wang J, Cai K, He X, Shen X, Wang J, Liu J, et al. Multiple-centre clinical evaluation of an ultrafast single-tube assay for SARS-CoV-2 RNA. Clin Microbiol Infect 2020;26(8):1076 - 81. http://dx.doi.org/ 10.1016/j.cmi.2020.05.007

11. Zheng YZ, Chen JT, Li J, Wu XJ, Wen JZ, Liu XZ, et al. Reverse transcription recombinase-aided amplification assay with lateral flow dipstick assay for rapid detection of 2019 novel coronavirus. Front Cell Infect Microbiol 2021;11:1-7. http://dx.doi.org/10.3389/fcimb. 2021.613304.

12. Wu T, Ge YY, Zhao KC, Zhu XJ, Chen Y, Wu B, et al. A reversetranscription recombinase-aided amplification assay for the rapid detection of $\mathrm{N}$ gene of severe acute respiratory syndrome coronavirus 2(SARS-CoV-2). Virology 2020;549:1 - 4. http://dx.doi.org/10.1016/ j.virol.2020.07.006.

13. Pan BH, Ji ZW, Sakkiah S, Guo WJ, Liu J, Patterson TA, et al. Identification of epidemiological traits by analysis of SARS-CoV-2 sequences. Viruses 2021;13(5):764. http://dx.doi.org/10.3390/ v13050764.

14. Onyeaka H, Anumudu CK, Al-Sharify ZT, Egele-Godswill E, Mbaegbu P. COVID-19 pandemic: a review of the global lockdown and its farreaching effects. Sci Prog 2021;104(2):368504211019854. http://dx. doi.org/10.1177/00368504211019854.

15. Yüce M, Filiztekin E, Özkaya KG. COVID-19 diagnosis -A review of current methods. Biosens Bioelectron 2021;172:112752. http://dx. doi.org/10.1016/j.bios.2020.112752. 